\title{
Desigualdades por SeXo e POR RaÇa E O diReito AOS TRANSPlanteS de ÓRgãos NO BRASIL
}

\author{
INEQUALITIES BY GENDER AND RACE AND THE RIGHT \\ TO ORGAN TRANSPLANTANTION IN BRAZIL
}

\author{
Alexandre Marinho* \\ Simone de Souza Cardoso** \\ Vivian Vicente de Almeida***
}

\section{RESUMO}

O presente texto trata do perfil dos transplantados e do problema das desigualdades no acesso aos transplantes de órgãos no Sistema Nacional de Transplantes (SNT) do Sistema Único de Saúde (SUS). A equidade nos procedimentos relacionados com transplantes de órgãos no Brasil recebe garantias constitucionais. Um recorte por gênero indica que o Brasil tem população com maioria de mulheres. Sob o ponto de vista étnico, pretos e pardos são a maioria da população. As necessidades de transplantes não diferem muito entre esses diferentes grupos populacionais. A despeito disso, contata-se que a maioria dos transplantes de órgãos, no Brasil, são feitos em homens e em brancos. Existem inúmeras causas para essas desigualdades.

\section{Palavras-chave:}

Desigualdade; Sistema Único de Saúde; Transplante de Órgãos.

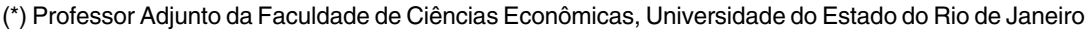
(UERJ). Técnico de Planejamento e Pesquisa do Instituto de Pesquisa Econômica Aplicada (IPEA). Rio de Janeiro/RJ - Brasil. E-mail: alexandre.marinho@ ipea.gov.br.

${ }^{(* *}$ ) Assistente de Pesquisa do Programa de Pesquisa para o Desenvolvimento Nacional (PNPD/ IPEA). Rio de Janeiro/RJ - Brasil.

${ }^{* * *}$ Assistente de Pesquisa do Programa de Pesquisa para o Desenvolvimento Nacional (PNPD/ IPEA). Rio de Janeiro/RJ - Brasil.

Texto recebido em 03.12.10. Revisado em 21.06.11. Aprovado em 22.06.11.
} 


\section{ABSTRACT}

According to Brazilian law, access to organ transplantation in Brazil should be equitable for all and not be influenced by race, geography, religion, or economic status. But we show that non-whites and women are underrepresented among organ transplantation recipients although they are no longer minorities in Brazil. The problem is reinforced, since there are not marked differences in the needs of organ transplantation against these groups. These inequalities may result from a number of factors.

\section{Keywords:}

Brazilian National Health System; Inequality; Organ Transplantation.

\section{INTRODUÇÃO}

O presente texto trata do perfil dos transplantados e do problema das desigualdades no acesso aos transplantes de órgãos no Sistema Nacional de Transplantes do Brasil (SNT) que, como veremos, têm recebido pouca atenção. Podemos especular que tal escassez investigativa também decorra da apenas recente inserção do direito à saúde no texto das constituições brasileiras com imperatividade direta aos órgãos públicos ${ }^{(1)}$. Especificamente, a análise será feita com base em indicadores relacionados aos receptores de transplantes discriminados por sexo e etnia. Restringiremos a nossa análise aos transplantes de rim e de fígado por razão de concisão. Mas as conclusões para outros órgãos (coração; pulmão; pâncreas; e transplante simultâneo de pâncreas e rim) estão disponíveis com os autores do presente trabalho, cujos resultados não diferem muito dos que serão aqui apresentados. Juntos, os transplantes de fígado e de rim correspondem a, aproximadamente, $90 \%$ das quantidades e $90 \%$ dos gastos com transplantes de órgãos sólidos no país.

De acordo com a página eletrônica do $\mathrm{SNT}^{(2)}$, o Brasil possui hoje um dos maiores programas públicos de transplantes de órgãos e tecidos do mundo. Com 548 estabelecimentos de saúde e 1.376 equipes médicas autorizados a realizar transplantes, o SNT está presente em 25 estados do país, por meio das Centrais Estaduais de Transplantes. A Política Nacional de Transplantes de órgãos e

(1) DALLARI, Sueli Gandolfi. A construção do direito à saúde no Brasil. Revista de Direito Sanitário, São Paulo, v. 9, n. 3, p. 9-34, nov. 2008/fev. 2009.

(2) Disponível em: <http://portal.saude.gov.br/portal/saude/area.cfm?id_area=1004>. Acesso em: 9 maio 2012. 
tecidos está fundamentada na legislação (Leis $n^{\circ} 9.434 / 97^{(3)}$ e $n^{\circ} 10.211 / 01^{(4)}$ ), tendo como diretrizes a gratuidade da doação, a beneficência em relação aos receptores e não maleficência em relação aos doadores vivos. Toda a política de transplante está em sintonia com as Leis $\mathrm{n}^{\circ} 8.080 / 90^{(5)}$ e $\mathrm{n}^{\circ} 8.142 / 90^{(6)}$, que regem o funcionamento do SUS.

Ainda de acordo com o SNT ${ }^{(7)}$, apenas no primeiro semestre do ano de 2009, foram realizados 8.192 transplantes dos seguintes órgãos e de córnea no país (coração: 96; córnea: 6.151; fígado/rim: 4; pulmão: 22; fígado: 602; pâncreas: 26; pâncreas/rim: 54; rim: 1.237). No mesmo ano, ainda de acordo com o SNT ${ }^{(8)}$, havia 63.866 pessoas nas listas de espera por transplantes de órgãos e de córnea no país (coração: 305; córnea: 23.756; fígado: 4.304; pâncreas: 124; pulmão: 164; rim: 34.640; rim/pâncreas: 576).

A inegável capacidade brasileira na realização de transplantes remove um possível óbice presente na prestação de cuidados à saúde da população. Dallari(9) (1988) assevera que "De fato, unicamente no Estado desenvolvido socioeconômica e culturalmente o indivíduo é livre para procurar um completo bem-estar físico, mental e social". Esse "completo bem-estar", que corresponde ao próprio conceito de saúde apregoado pela Organização Mundial da Saúde (OMS), depende, portanto, de condições objetivas, pois "enquanto direito coletivo, a saúde depende igualmente do estágio de desenvolvimento do Estado"(10). $\mathrm{O}$ Estado brasileiro reúne as condições potenciais necessárias para assegurar o equânime direito aos transplantes para os que deles necessitarem. Mas, conforme veremos, tais condições ainda não se materializaram.

A Constituição Brasileira estabelece que a saúde é "um direito de todos e um dever do Estado", o qual deveria garantir o "acesso universal igualitário

(3) BRASIL. Lei 9.434, de 4 de fevereiro de 1997. Dispõe sobre a remoção de órgãos, tecidos e partes do corpo humano para fins de transplante e tratamento e dá outras providências. Disponível em: <http://www.planalto.gov.br/ccivil_03/LEIS/L9434compilado.htm>. Acesso em: 2 jul. 2012.

(4) BRASIL. Lei 10.211, de 23 de março de 2001. Altera dispositivos da Lei no 9.434 , de 4 de fevereiro de 1997, que dispõe sobre a remoção de órgãos, tecidos e partes do corpo humano para fins de transplante e tratamento. Disponível em: <http://www.planalto.gov.br/ccivil_03/Leis/LEIS_2001/ L10211.htm>. Acesso em: 2 jul. 2012.

(5) BRASIL. Lei $n^{\circ} 8.080$, de 19 de setembro de 1990, Dispõe sobre as condições para a promoção, proteção e recuperação da saúde, a organização e o funcionamento dos serviços correspondentes e dá outras providências. Disponível em: <http://www.planalto.gov.br/ccivil_03/Leis/L8080.htm>. Acesso em: 12 jul. 2012.

(6) BRASIL. Lei $n^{\circ} 8.142$, de 28 de dezembro de 1990. Dispõe sobre a participação da comunidade na gestão do Sistema Único de Saúde (SUS) e sobre as transferências intergovernamentais de recursos financeiros na área da saúde e dá outras providências. Disponível em: <http://www.planalto. gov.br/ccivil_03/LEIS/L8142.htm>. Acesso em: 12 jul. 2012.

(7) Disponível em: <http://portal.saude.gov.br/portal/arquivos/pdf/TRANSPLANTES_2009.pdf>. Acesso em: 7 out. 2010.

(8) Disponível em: <http://portal.saude.gov.br/portal/arquivos/pdf/Lista_de_Espera_2009.pdf>.

Acesso em: 7 out. 2010.

(9) DALLARI, Sueli Gandolfi. O direito à saúde. Revista de Saúde Pública, São Paulo, v. 22, n. 1, p.57-63, 1988.

(10) Id. Ibid. 
às ações e serviços" de saúde (Art. 196). Dallari(11) ressalta que a Constituição Federal também regula explicitamente os transplantes de órgãos e tecidos em seu Art. $199 \S 4^{\circ}$ ditando: "A lei disporá sobre as condições e os requisitos que facilitem a remoção de órgãos, tecidos e substâncias humanas para fins de transplante, pesquisa e tratamento, bem como a coleta, processamento e transfusão de sangue e seus derivados, sendo vedado todo tipo de comercialização". Dessa forma, de acordo com a legislação, todos os potenciais beneficiários de transplantes devem estar inscritos em lista única de espera, dentre os quais serão escolhidos os que receberão o transplante, conforme critérios estabelecidos para cada órgão ou tecido, incluindo a compatibilidade entre doador e receptor, e o tempo decorrido da inscrição na lista única. Além disso, são estabelecidos, para cada tipo de órgão, os critérios técnicos de urgência. Assim, o texto constitucional e a legislação pertinente excluem os mecanismos de mercado e corroboram a garantia de oferta gratuita de cuidados à saúde a todos que dela necessitam, o que, nas palavras de Nogueira e Pires ${ }^{(12)}$, "corresponde ao ideal de igualdade e que, por sua vez, se submete ao pleno desenvolvimento do Estado democrático de direito".

A literatura revela muitas causas para desigualdades em transplantes: fatores biológicos; condições de saúde dos pacientes; características socioeconômicas gerais; questões culturais; a atuação das instituições de procura de órgãos, dos centros transplantadores e dos sistemas de saúde nos quais elas se inserem; a posse de planos de saúde privados; a relativa escassez de pesquisas destinadas às minorias; e, de modo não conclusivo, procedimentos ou sentimentos discriminatórios e racismo. O caso brasileiro não estaria, a priori, isento de tais percalços. Assim, nosso texto também objetiva a desejável avaliação da existência, ou não, da necessária "igualdade da cidadania em face das questões sanitárias"(13), já que o Estado brasileiro tem a capacidade para a realização de transplantes em larga escala. Entretanto, ao menos no nível regional, os transplantes são feitos de modo desigual, e ainda intempestivo, no território brasileiro(14), embora a igualdade de acesso seja um imperativo constitucional dotado de aparato regulatório potencialmente eficaz. Conforme Dallari ${ }^{(15)}$,

Com efeito, diferentemente dos demais direitos sociais ali afirmados, apenas o direito à saúde tem sua garantia claramente vinculada às políticas sociais e econômicas, as diretrizes do sistema expressamente formuladas, envolvendo a participação da comunidade, e suas atribuições enumeradas no próprio texto constitucional (CF/88, arts. 196, 198 e 200).

(11) DALLARI, op. cit., 2009.

(12) NOGUEIRA, Vera Maria Ribeiro; PIRES, Denise Elvira Pereira. Direito à saúde: um convite à reflexão. Cadernos de Saúde Pública, Rio de Janeiro, v. 20, n. 3, p. 753-760, maio-jun. 2004.

(13) Id. Ibid.

(14) MARINHO, Alexandre; CARDOSO, Simone de Souza; ALMEIDA, Vivian Vicente de. Disparidades nas filas de transplantes de órgãos nos estados brasileiros. Cadernos de Saúde Pública, Rio de Janeiro, v. 26, n. 4 , p. $786-796$, abr. 2010.

(15) Id. Ibid. 
Este texto, além desta Introdução e de nossas Considerações Finais, é composto por mais cinco seções. Na seção I fazemos uma breve análise da literatura existente sobre o tema no Brasil e no exterior. Na seção II mostramos uma caracterização simplificada da demografia brasileira por sexo e por raça, com as implicações sobre os transplantes de órgãos no país. Na seção III consta uma análise preliminar da mortalidade por causas externas no Brasil. Na seção IV fazemos uma avaliação dos transplantes de fígado e de rim no Brasil, enfatizando a segmentação por sexo e por raça.

\section{BREVE EXAME DA LITERATURA}

A literatura internacional sobre desigualdade de acesso e de resultados nos transplantes de órgãos é ampla. Nos Estados Unidos, a Joint Commission on Accreditation of Healthcare Organizations ${ }^{(16)}$ reporta que, embora os afro-americanos representassem apenas $12,3 \%$ da população americana, eles representavam $35 \%$ das pessoas na lista de espera por doação de rim. Pacientes brancos esperariam em média 675 dias para receber um transplante de rim, enquanto pacientes negros esperariam em média 1.603 dias. Os afro-americanos teriam menos da metade de probabilidade que os brancos têm de receber um transplante de rim. Seus enxertos têm taxas de sobrevivência após cinco anos de transplantes entre 10\% e $20 \%$ menores do que dos brancos. E receberiam, apenas, $14 \%$ de doações intervivos, enquanto os brancos recebem $78 \%$ desse tipo de doação. Ainda na JCAHO, reporta-se que "Equity in the organ allocation and transplantation equation has yet to be achieved"(17). As desigualdades em transplantes de órgãos são relatadas, em muitos outros trabalhos e em diversos países. Entre outros, destacamos: Roodnat et $a^{(18)}$, Kurz et a(19), Myaskovsky et a(20), Rudge et a( ${ }^{(21)}$, Higgins

(16) JOINT COMMISSION ON ACCREDITATION OF HEALTHCARE ORGANIZATIONS - JCAHO. Health care at crossroads: strategies for narrowing the organ donation gap and protecting patients. Washington, DC, 2004.

(17) Id. Ibid.

(18) ROODNAT, Joke I.; VAN DE WETERING, Jacqueline; ZUIDEMA, Willij; VAN NOORD, Marian A. A.; KAL-VAN GESTEL, Judith A.; IJZERMANS, Jan N. M.; WEIMAR, Willem. Ethnically diverse populations and their participation in living donation programs. Transplantation, Baltimore, v. 89, n.10, p. 1263-1269, May 2010.

(19) KURZ, Richard S.; SCHARFF, Darcell P.; TERRY, Tanchica; ALEXANDER, Shanica; WATERMAN, Amy. Factors influencing organ donation decisions by African Americans: a review of the literature. Medical Care Research and Review, Thousand Oaks, v. 64, n. 5, p. 475-517, Oct. 2007.

(20) MYASKOVSKY, Larissa; SWITZER, Galen E.; CROWLEY-MATOKA, Megan; UNRUH, Mark; DiMARTINI, Andrea F.; DEW, Mary Amanda. Psychosocial factors associated with ethnic differences in transplantation. Current Opinion in Organ Transplantation, New York, v. 12, n. 2, p. 182-187, Apr. 2007. (21) RUDGE, Chris; JOHNSON, Rachel J.; FUGGLE, Susan V.; FORSYTHE, John L. R. Renal transplantation in the United Kingdom for patients from ethnic minorities. Transplantation, Baltimore, v. 83 , n. 9, p. 1169-1173, May 2007. 
e Fishman (22), Simmerling ${ }^{(23)}$, Gordon et $a^{(24)}$, Alexander et $a^{(25)}$, Beasley et $a^{(26)}$, Callender ${ }^{(27)}$ e Ozminkowski et a/(28).

No Brasil, entretanto, a abordagem desse importante tema é limitada. Desigualdades gerais em saúde, com melhor situação de homens e brancos, são reportadas em Noronha e Andrade(29). Já, para os transplantes de órgãos, Macha$d o^{(30)}$ estuda os 835 pacientes inscritos na lista de espera para transplante renal em Belo Horizonte, no estado de Minas Gerais, entre os anos 2000 e 2005. Foi observado que 520 (62,3\%) dos pacientes em lista eram do sexo masculino e 315 $(37,7 \%)$ eram do sexo feminino. Do total, $46,1 \%$ eram brancos, e $51,9 \%$ pertenciam a outras etnias. Entre os 190 pacientes transplantados, ao final do período, 119 $(62,6 \%)$ eram homens e $71(37,4 \%)$ eram mulheres. Ainda entre os transplantados, $82(50,3 \%)$ eram brancos, $81(49,7 \%)$ eram não brancos. Gomes ${ }^{(31)}$ aponta que são necessárias, para melhor equidade no SNT, correções normativas, especificação clara de princípios de justiça e de critérios de alocação, aperfeiçoamento gerencial, planejamento estratégico, transparência de informações e abordagem sistêmica. Garcia et $a^{(32)}$ afirmam que "Equidade significa imparcialidade e que,... portanto, a etnia, cor ou o credo não devem desempenhar nenhum papel na alocação de órgãos". Entretanto, reconhecem a existência de barreiras aos transplantes de

(22) HIGGINS, R. S. D.; FISHMAN, J. A. Disparities in solid organ transplantation for ethnic minorities: facts and solutions. American Journal of Transplantation, Copenhagen, v. 6, n. 11, p. 2556-2562, 2006. (23) SIMMERLING, Mary. The case of the national deceased organ donor waiting list as a measure of equity of access to organ transplantation. ICFAI Journal of Healthcare Law, Dehradun, v. 4, n. 3 , p. 15-24, 2006.

(24) GORDON, Elisa J.; PROHASKA, Thomas; SIMINOFF, Laura A.; MINICH, Peter J.; SEHGAL, Ashwini R. Can focusing on self-care reduce disparities in kidney transplant outcomes? American Journal of Kidney Diseases, Philadelphia, v. 45, n. 5, p. 935-940, May 2005.

(25) ALEXANDER, G. Caleb; SEHGAL, Ashwini. Barriers to cadaveric renal transplantation among blacks, women, and the poor. JAMA, Chicago, v. 280, n. 13, p. 1148-1152, Oct. 1998.

(26) BEASLEY, C.; GUADAGNOLI, E.; MCNAMARA, P.; EVANISKO, M. Organ donation and ethnicity: predicting support for organ donation among ethnic minorities. Transplantation, v. 65, n. 5, Supplement, p. 95, 1998.

(27) CALLENDER, C. O. Obstacles to organ donation in ethnic minorities. Pediatric Transplantation, Copenhagen, v. 5, n. 6, p. 383-385, 2001.

(28) OZMINKOWSKY, Ronald J.; WHITE, Alan J.; HASSOL, Andrea; MURPHY, Michael. Minimizing racial disparity regarding receipt of a cadaver kidney transplant. American Journal of Kidney Diseases, Philadelphia, v. 30, n. 6, p. 749-759, Dec. 1997.

(29) NORONHA, Kenya Valéria Micaela de Souza; ANDRADE, Monica Viegas. O efeito da distribuição de renda sobre o estado de saúde individual no Brasil. Pesquisa e Planejamento Econômico, Brasília, v. 37, n. 3, p. 521-556, dez. 2007.

(30) MACHADO, Elaine Leandro. Equidade no acesso ao transplante renal em Belo Horizonte, 20002005. 2007. 120 f. Dissertação (Mestrado em Saúde Pública) - Faculdade de Medicina da Universidade Federal de Minas Gerais, Belo Horizonte, 2007.

(31) GOMES, F. B. C. Ameaças à equidade na distribuição de órgãos para transplante no Brasil: uma análise dos critérios legais de acesso. Brasília: Departamento de Serviço Social, Instituto de Ciências Humanas, Universidade de Brasília, 2007.

(32) GARCIA, Valter Duro; ABBUD FILHO, Mario; PESTANA, J. O. M. Alocação de órgãos e tecidos. In: GARCIA, Valter Duro; ABBUD FILHO, Mario; NEUMANN, Jorge; PESTANA, J. O. M. Transplante de órgãos e tecidos. 2 ed. São Paulo: Segmento Farma, 2006. p. 158-173. 
rim em minorias. Marinho et $a^{(33)}$ apontam disparidades significativas nos tempos de espera e na produção de transplantes de órgãos entre os estados brasileiros. Junqueira et $a^{(34)}$ relata que os homens $(56,9 \%)$ e os brancos $(62,7 \%)$ foram maioria entre os doadores de pulmão no Estado de São Paulo no ano de 2006. Barcellos ${ }^{(35)}$, pesquisando indivíduos maiores de 20 anos de idade na zona urbana de Pelotas (RS), encontra maior disposição para doar órgãos em indivíduos jovens, brancos e com escolaridade e renda elevadas.

\section{UM RETRATO DEMOGRÁFICO SIMPLIFICADO DO BRASIL}

Nesta seção explicitamos as proporções relativas ao sexo e à cor ou raça e como esses grupos estão representados na população brasileira. De acordo com o Instituto Brasileiro de Geografia e Estatística (IBGE)(36), a população residente no Brasil é composta, atualmente, por 189.953 .000 brasileiros. Esse contingente populacional é dividido em, aproximadamente, 92.433 .000 homens e 97.520 .000 mulheres. Em termos percentuais temos $49 \%$ de homens e $51 \%$ de mulheres. A população brasileira apresenta $48,4 \%$ de sua população branca, $6,8 \%$ preta, $43,8 \%$ parda e $0,9 \%$ indígena. Mais de $50 \%$ da população brasileira é, portanto, composta por não brancos. Na Tabela 1 mostramos a proporção de homens e mulheres com relação à cor ou raça.

\begin{tabular}{|lccc|}
\hline \multicolumn{4}{|c|}{ Tabela 1 - Distribuição percentual das pessoas por sexo, segundo cor ou raça } \\
\hline POPULAÇÃO POR RAÇA E SEXO - BRASIL, 2008 & Homens & Mulheres & Total \\
\hline Indígena & 0,13 & 0,15 & 0,28 \\
Branca & 23,03 & 25,40 & 48,43 \\
Preta & 3,40 & 3,44 & 6,84 \\
Amarela & 0,26 & 0,32 & 0,58 \\
Parda & 21,80 & 22,00 & 43,80 \\
Sem declaração & 0,03 & 0,03 & 0,07 \\
\hline Total & 48,66 & 51,34 & 100,00 \\
\hline
\end{tabular}

Fonte: IBGE/PNAD de 2008.

(33) MARINHO, op. cit.

(34) FERNANDES, Paulo Manuel Pêgo; SAMANO, Marcos Naoyuki; JUNQUEIRA, Jader Joel Machado; WAISBERG, Daniel Reis; NOLETO, Gustavo Sousa; JATENE, Fabio Biscegli. Perfil do doador de pulmão disponibilizado no estado de São Paulo, Brasil, em 2006. Jornal Brasileiro de Pneumologia, Brasília, v. 34, n. 7, p. 497-505, jul. 2008.

(35) BARCELLOS, Franklin Correa. Intenção de doar órgãos em uma população adulta. 2003. 189 f. Dissertação (Mestrado em Epidemiologia) - Faculdade de Medicina da Universidade Federal de Pelotas, Pelotas, 2003.

(36) INSTITUTO BRASILEIRO DE GEOGRAFIA E ESTATÍSTICA - IBGE. Síntese de indicadores sociais. Rio de Janeiro, 2009. 


\section{CAUSAS EXTERNAS - UM ASPECTO DA DESIGUALDADE}

Nesta parte do estudo, vamos cuidar da exposição de um dado fundamental para o sistema de saúde brasileiro e que pode ter um impacto significativo no sistema de transplantes: as mortes por causas externas, que potencializam os quantitativos disponíveis de órgãos para transplantes provenientes de pessoas saudáveis e jovens. Segundo o Datasus, no ano de 2007, aproximadamente 131.032 pessoas morreram por causas externas no Brasil, e a população masculina responde por mais de $80 \%$ dessas mortes. $\mathrm{Na}$ Tabela 2 são mostrados os valores das mortalidades por causas externas para homens e mulheres.

\begin{tabular}{|lcc|}
\hline \multicolumn{3}{|l|}{ Tabela 2 - Proporção de óbitos por causas externas segundo o sexo (2007) } \\
\hline \multicolumn{2}{|l|}{ Mortalidade por Causas Externas } & $\%$ \\
\hline Sexo & Total & 83,43 \\
Homens & 109.323 & 16,49 \\
Mulheres & 21.605 & 100,00 \\
\hline Total & 131.032 & \\
\hline
\end{tabular}

Fonte: Datasus

Quando observada a mortalidade desagregada por cor ou raça, na Tabela 3 , abaixo, vemos que os brancos respondem por aproximadamente $41 \%$ da mortalidade por causas externas, enquanto pretos e pardos, somados, atingem a maioria das mortes por essa causa, aproximadamente $51 \%$.

Tabela 3 - Proporção de óbitos por causas externas segundo cor e raça (2007)

\begin{tabular}{|lcc|}
\hline Mortalidade por Causas Externas & & \\
\hline Cor/raça & Total & $\%$ \\
Branca & 54.742 & 41,78 \\
Preta & 8.894 & 6,79 \\
Parda & 58.984 & 45,01 \\
Outros & 8.412 & 6,42 \\
\hline Total & 131.032 & 100,00 \\
\hline
\end{tabular}

Fonte: Datasus 
O exame da literatura indica que, em vários países, a desigualdade nos transplantes pode ser explicada pela insuficiência de doadores com potenciais compatibilidades para minorias raciais. No caso brasileiro, entretanto, a quantidade de mortes por causas externas incide, preponderantemente, sobre a população não branca, o que evidenciaria uma expressiva quantidade de potenciais doadores para este grupo populacional.

\section{GÊNERO, RAÇA E TRANSPLANTES DE ÓRGÃOS NO BRASIL}

A investigação com relação aos receptores de transplantes no Brasil será feita, principalmente, com base nos dados fornecidos pela Associação Brasileira de Transplantes de Órgãos ${ }^{(37)}$. Essa análise será desagregada por sexo e por cor.

Fígado: o fígado é o órgão com maior custo unitário para transplante no país (custo de $R \$ 57.098,41$ para doador morto e de $R \$ 57.939,71$ para doador vivo). Tal órgão representa, aproximadamente, $55 \%$ dos gastos com órgãos sólidos no ano de 2010 e tem um prazo médio de espera de 185 dias, de acordo com o SNT. Foram transplantados 602 fígados no primeiro semestre de 2010, ou seja, quase $30 \%$ dos 2.037 órgãos sólidos transplantados no período, de acordo com o SNT. A seguir, na Figura 1, vemos uma representação das doenças que indicam transplante de fígado, de acordo com a ABTO.

Figura 1 - Proporção das morbidades potencialmente indicativas para transplantes de fígado $(2007)^{(38)}$

\section{Indicação para transplante}

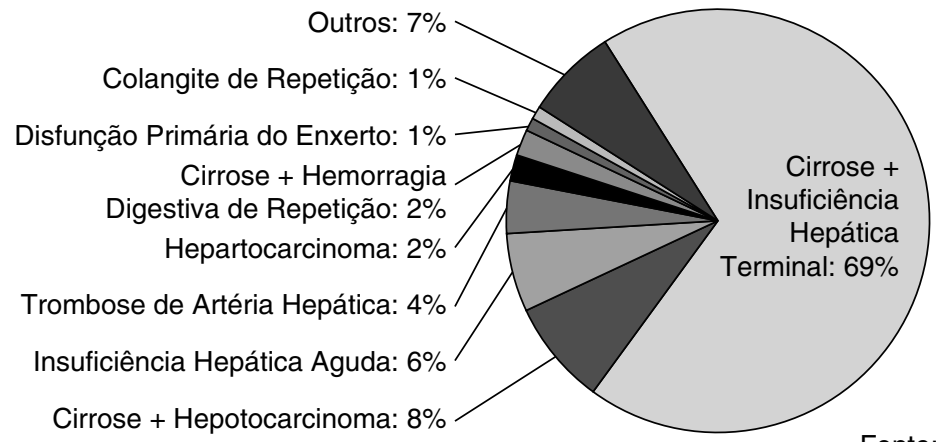

Fonte: ABTO, 2007

(37) A análise da ABTO contempla o período de 1995 a 2004. ASSOCIAÇÃO BRASILEIRA DE TRANSPLANTES DE ÓRGÃOS - ABTO. Registro brasileiro de transplantes: edição comemorativa: 10 Anos: análise qualitativa. São Paulo, 2007.

(38) Id. Ibid. 
Observamos que aproximadamente $80 \%$ das indicações de transplante são em função de diagnósticos de cirrose. A seguir, na Tabela 4, vemos a proporção da mortalidade por cirrose em função da cor ou raça.

Tabela 4 - Proporção de óbitos por fibrose e cirrose do fígado segundo cor e raça (2007)

\begin{tabular}{|lc|}
\hline Mortalidade - Fibrose e cirrose do fígado - Ano 2007 & Total \\
\hline Branca & 57,45 \\
Preta & 7,49 \\
Parda & 35,06 \\
\hline Total & 100,00
\end{tabular}

Fonte: Datasus - Indicadores de Saúde

A maioria $(57,45 \%)$ da mortalidade se concentra na população branca. Mas esse valor é bem menor que a proporção apresentada para receptores de cor branca $(81 \%)$ que vemos na Figura 2, a seguir, evidenciando uma possível desigualdade racial na recepção de fígados no país.

Figura 2 - Proporção das pessoas receptoras do órgão fígado segundo cor ou raça $(2007)^{(39)}$

\section{Raça do receptor}

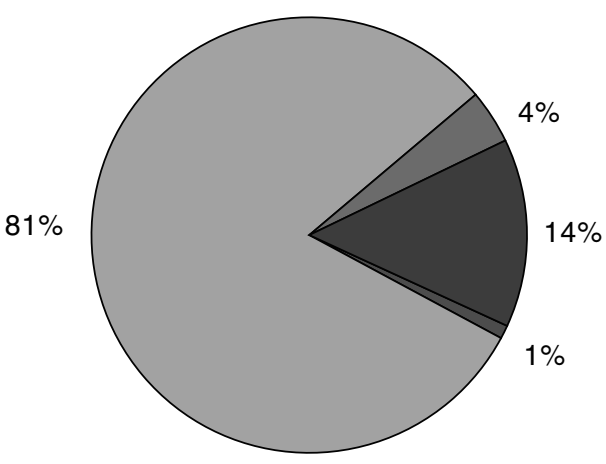

Fonte: ABTO, 2007

(39) Id. Ibid. 
Na Figura 3, a seguir, observamos uma preponderância dos homens (63\%) como receptores de fígado, em relação às mulheres, o que poderia ser justificável, em função da maior incidência de cirrose entre os homens ${ }^{(40)}$.

Figura 3 - Proporção das pessoas receptoras do órgão fígado segundo o sexo $(2007)^{(41)}$

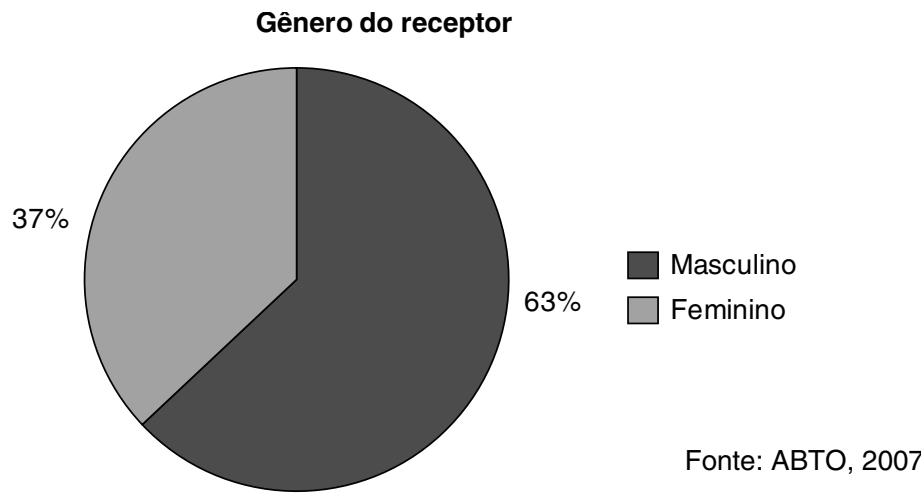

Rim: Somente no primeiro semestre do ano de 2009 foram realizados 1.237 transplantes de rim no país, ou seja, quase $60 \%$ do total de 2.037 transplantes de órgãos sólidos transplantados no período. O custo médio de um transplante de rim com doador vivo é de $\mathrm{R} \$ 15.559,58$ e $\mathrm{R} \$ 20.236,39 \mathrm{com}$ doador falecido, implicando em, aproximadamente, $35 \%$ dos gastos com transplantes de órgãos sólidos no ano de 2010. O rim tem o maior tempo de espera para transplante, de acordo com o SNT (837 dias).

Os homens são $45 \%$ dos casos e as mulheres são $55 \%$ dos casos de insuficiência renal crônica (IRC) no Brasil, conforme a Tabela 5, a seguir.

Tabela 5 - Percentual dos portadores de IRC declarado por algum médico ou profissional de saúde

\begin{tabular}{|lcc|}
\hline Gênero & Frequência & Percentagem \\
\hline Masculino & 2.143 & 45 \\
Feminino & 2.618 & 55 \\
\hline Total & 4.761 & 100 \\
\hline
\end{tabular}

Fonte: IBGE/PNAD 2008

(40) LAURENTI, Ruy; JORGE, Maria Helena Prado de Mello; GOTLIEB, Sabina Léa Davidson. Perfil epidemiológico da morbi-mortalidade masculina. Ciência \& Saúde Coletiva, Rio de Janeiro, v. 10, n. 1, mar. 2005. Disponível em: <http://www.scielo.br/scielo.php?script=sci_arttext\&pid=S1413$81232005000100010 \&$ lng=pt\&nrm=iso >. Acesso em: 9 maio 2012.

(41) ASSOCIAÇÃO, op. cit. 
Entretanto, os homens são $61 \%$ dos pacientes que receberam transplantes de rim, conforme a Figura 4, também a seguir.

Figura 4 - Proporção das pessoas receptoras de rim segundo o sexo (2007) $)^{(42)}$

\section{Gênero do receptor}

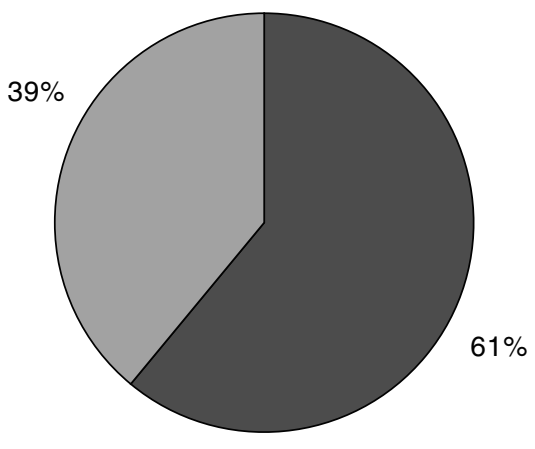

Masculino

Feminino

Fonte: ABTO, 2007

Conforme a Tabela 6, a seguir, os indivíduos de raça branca são a minoria $(48,2 \%)$ dos portadores de IRC. Mas, observamos na Figura 5 , também a seguir, uma predominância (69\%) da cor branca entre os receptores de rim.

Tabela 6 - Percentual dos portadores de insuficiência renal crônica declarada por algum médico ou profissional de saúde

\begin{tabular}{|lcc|}
\hline Cor ou Raça & Frequência & Percentagem \\
\hline Indígena & 25 & 0,5 \\
Branca & 2.297 & 48,2 \\
Preta & 335 & 7,0 \\
Amarela & 23 & 0,5 \\
Parda & 2.078 & 43,6 \\
Sem declaração & 3 & 0,1 \\
\hline Total & 4.761 & 100,0 \\
\hline
\end{tabular}

Fonte: IBGE/PNAD 2008

(42) Id. Ibid. 
Figura 5 - Proporção das pessoas receptoras do órgão rim segundo cor ou raça $(2007)^{(43)}$

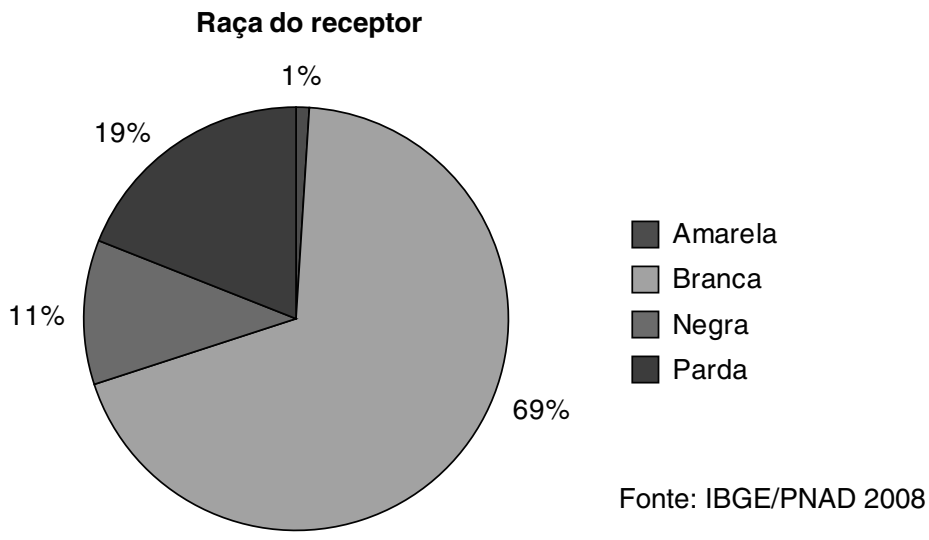

\section{CONSIDERAÇÕES FINAIS}

A Constituição Federal Brasileira de 1988, em seu Art. 196, estabelece que

A saúde é direito de todos e dever do Estado, garantido mediante políticas sociais e econômicas que visem à redução do risco de doença e de outros agravos e ao acesso universal e igualitário às ações e serviços, para a sua promoção, proteção e recuperação.

O acesso igualitário aos transplantes nos parece, entretanto, uma garantia constitucional ainda não atendida. Seria forçoso reconhecer, como afirma Dallari ${ }^{(44)}$, que: "Tem-se, portanto, um largo caminho a percorrer para que o direito à saúde afirmado em 1988 seja uma realidade para todos no Brasil".

Vimos que existem mais brasileiras que brasileiros e que o Brasil não é mais um país de maioria branca. Contudo, constatamos que a grande maioria dos receptores de transplantes de rim e de fígado são homens. Também verificamos que a maior parte dos receptores desses órgãos são brancos. Contudo, tanto as mulheres quanto os pretos e os pardos apresentam necessidades bem semelhantes às dos homens brancos.

No presente estudo, não conseguimos encontrar justificativas no campo demográfico, ou no epidemiológico, que justifiquem o descumprimento aparente

(43) Ibid.

(44) DALLARI, op. cit., 2009. 
das diretrizes imperativas da Carta Magna, embora reconheçamos a modernidade da presença do direito à saúde na história constitucional brasileira e que, conforme adverte Dallari"45): "É evidente, entretanto, que essa contemporaneidade do direito à saúde, tal qual construído na Constituição brasileira de 1988, traz consigo sérias dificuldades".

As razões para essas desigualdades de gênero e de etnias são muitas e complexas. Preconceitos; medos; desinformação; biologia humana; subfinanciamento da saúde; racismo; e vieses desfavoráveis às mulheres e às minorias contribuem para as desigualdades nos transplantes de órgãos no Brasil e no exterior.

O problema das desigualdades em transplantes em nosso país deve ser assumido com transparência e franqueza democráticas, com a compreensão de que ele não é um fenômeno natural, nem fortuito, nem irredutível. Como, no Brasil, os mecanismos de regulação via mercado são liminarmente descartados na seara dos transplantes, é imperiosa a tarefa de busca de aperfeiçoamentos das políticas públicas de saúde pertinentes ao tema.

\section{REFERÊNCIAS}

ALEXANDER, G. Caleb; SEHGAL, Ashwini. Barriers to cadaveric renal transplantation among blacks, women, and the poor. JAMA, Chicago, v. 280, n. 13, p. 1148-1152, Oct. 1998.

ASSOCIAÇÃO BRASILEIRA DE TRANSPLANTES DE ÓRGÃOS - ABTO. Registro brasileiro de transplantes: edição comemorativa: 10 Anos: análise qualitativa. São Paulo, 2007.

BARCELLOS, Franklin Correa. Intenção de doar órgãos em uma população adulta. 2003. 189 p. Dissertação (Mestrado em Epidemiologia) - Faculdade de Medicina da Universidade Federal de Pelotas, Pelotas, 2003.

BEASLEY, C.; GUADAGNOLI, E.; MCNAMARA, P.; EVANISKO, M. Organ donation and ethnicity: predicting support for organ donation among ethnic minorities. Transplantation, v. 65, n. 5, Supplement, p. 95, 1998.

CALLENDER, C. O. Obstacles to organ donation in ethnic minorities. Pediatric Transplantation, Copenhagen, v. 5, n. 6, p. 383-385, 2001.

DALLARI, Sueli Gandolfi. O direito à saúde. Revista de Saúde Pública, São Paulo, v. 22, n. 1, p. 57-63, 1988.

A construção do direito à saúde no Brasil. Revista de Direito Sanitário, São Paulo, v. 9, n. 3, p. 9-34, nov. 2008/fev. 2009.

(45) Id. Ibid. 
FERNANDES, Paulo Manuel Pêgo; SAMANO, Marcos Naoyuki; JUNQUEIRA, Jader Joel Machado; WAISBERG, Daniel Reis; NOLETO, Gustavo Sousa; JATENE, Fabio Biscegli. Perfil do doador de pulmão disponibilizado no estado de São Paulo, Brasil, em 2006. Jornal Brasileiro de Pneumologia, Brasília, v. 34, n. 7, p. 497-505, jul. 2008.

GARCIA, Valter Duro; ABBUD FILHO, Mario; PESTANA, J. O. M. Alocação de órgãos e tecidos. In: GARCIA, Valter Duro; ABBUD FILHO, Mario; NEUMANN, Jorge; PESTANA, J. O. M. Transplante de órgãos e tecidos. 2. ed. São Paulo: Segmento Farma, 2006. p. 158-173.

GOMES, F. B. C. Ameaças à equidade na distribuição de órgãos para transplante no Brasil: uma análise dos critérios legais de acesso. Brasília: Departamento de Serviço Social, Instituto de Ciências Humanas, Universidade de Brasília, 2007.

GORDON, Elisa J.; PROHASKA, Thomas; SIMINOFF, Laura A.; MINICH, Peter J.; SEHGAL, Ashwini R. Can focusing on self-care reduce disparities in kidney transplant outcomes? American Journal of Kidney Diseases, Philadelphia, v. 45, n. 5, p. 935-940, May 2005.

HIGGINS, R. S. D.; FISHMAN, J. A. Disparities in solid organ transplantation for ethnic minorities: facts and solutions. American Journal of Transplantation, Copenhagen, v. 6, n. 11, p. 2556-2562, 2006.

INSTITUTO BRASILEIRO DE GEOGRAFIA E ESTATÍSTICA - IBGE. Síntese de indicadores sociais. Rio de Janeiro, 2009. Janeiro, 2008.

Pesquisa Nacional por Amostra de Domicílios - PNAD. Rio de Indicadores sociodemográficos e de saúde. Rio de Janeiro, 2009.

JOINT COMMISSION ON ACCREDITATION OF HEALTHCARE ORGANIZATIONS - JCAHO. Health care at crossroads: strategies for narrowing the organ donation gap and protecting patients. Washington, DC, 2004.

KURZ, Richard S.; SCHARFF, Darcell P.; TERRY, Tanchica; ALEXANDER, Shanica; WATERMAN, Amy. Factors influencing organ donation decisions by African Americans: a review of the literature. Medical Care Research and Review, Thousand Oaks, v. 64, n. 5, p. 475-517, Oct. 2007.

LAURENTI, Ruy; JORGE, Maria Helena Prado de Mello; GOTLIEB, Sabina Léa Davidson. Perfil epidemiológico da morbi-mortalidade masculina. Ciência \& Saúde Coletiva, Rio de Janeiro, v. 10, n. 1, mar. 2005. Disponível em <http://www.scielo.br/scielo.php?script=sci_arttext\&pid=S1413$81232005000100010 \& \mathrm{lng}=\mathrm{pt} \& \mathrm{nrm}=\mathrm{iso}>$. Acesso em: 9 maio 2012. 
MACHADO, Elaine Leandro. Equidade no acesso ao transplante renal em Belo Horizonte, 2000-2005. 2007. 120 p. Dissertação (Mestrado em Saúde Pública) - Faculdade de Medicina da Universidade Federal de Minas Gerais, Belo Horizonte, 2007.

MARINHO, Alexandre; CARDOSO, Simone de Souza; ALMEIDA, Vivian Vicente de. Disparidades nas filas de transplantes de órgãos nos estados brasileiros. Cadernos de Saúde Pública, Rio de Janeiro, v. 26, n. 4, p. 786-796, abr. 2010.

MYASKOVSKY, Larissa; SWITZER, Galen E.; CROWLEY-MATOKA, Megan; UNRUH, Mark; DiMARTINI, Andrea F.; DEW, Mary Amanda. Psychosocial factors associated with ethnic differences in transplantation. Current Opinion in Organ Transplantation, New York, v. 12, n. 2, p. 182-187, Apr. 2007.

NOGUEIRA, Vera Maria Ribeiro; PIRES, Denise Elvira Pereira. Direito à saúde: um convite à reflexão. Cadernos de Saúde Pública, Rio de Janeiro, v. 20, n. 3, p. 753-760, maio-jun. 2004.

NORONHA, Kenya Valéria Micaela de Souza; ANDRADE, Monica Viegas. O efeito da distribuição de renda sobre o estado de saúde individual no Brasil. Pesquisa e Planejamento Econômico, Brasília, v. 37, n. 3, p. 521-556, dez. 2007.

OZMINKOWSKY, Ronald J.; WHITE, Alan J.; HASSOL, Andrea; MURPHY, Michael. Minimizing racial disparity regarding receipt of a cadaver kidney transplant. American Journal of Kidney Diseases, Philadelphia, v. 30, n. 6, p. 749-759, Dec. 1997.

ROODNAT, Joke I.; VAN DE WETERING, Jacqueline; ZUIDEMA, Willij; VAN NOORD, Marian A. A.; KAL-VAN GESTEL, Judith A.; IJZERMANS, Jan N. M.; WEIMAR, Willem. Ethnically diverse populations and their participation in living donation programs. Transplantation, Baltimore, v. 89, n. 10, p. 1263-1269, May 2010.

RUDGE, Chris; JOHNSON, Rachel J.; FUGGLE, Susan V.; FORSYTHE, John L. R. Renal transplantation in the United Kingdom for patients from ethnic minorities. Transplantation, Baltimore, v. 83, n. 9, p. 1169-1173, May, 2007.

SIMMERLING, Mary. The case of the national deceased organ donor waiting list as a measure of equity of access to organ transplantation. ICFAI Journal of Healthcare Law, Dehradun, v. 4, n. 3, p. 15-24, 2006. 